\title{
Guest Editorial: Fractional-Order Circuits and Systems: Theory, Design, and Applications
}

\author{
Costas Psychalinos $^{1}$ - Ahmed S. Elwakil ${ }^{2,3}$. \\ Ahmed G. Radwan ${ }^{3,4}$ - Karabi Biswas ${ }^{5}$
}

Published online: 14 March 2016

(C) Springer Science+Business Media New York 2016

Nowadays, there is a significant research interest in the area of fractional-order circuits. This originates from the fact that they find applications in biochemistry, medicine, electrical engineering, and many other fields. For example, the modeling of viscoelasticity as well as of biological cells and tissues has been performed through the utilization of fractional-order calculus. This special issue is focused on the theory, design, and applications of fractional-order circuits and systems, with the purpose of offering to the circuits and systems community the opportunity to explore recent advances in fractional-order circuits and systems theory and design as well as of their applications.

A total of 20 papers have been accepted in this special issue and are arranged to cover the following subjects: circuit theory of fractional-order circuits, fractional-order filter and oscillator design and applications, digital circuits and systems approximating

Costas Psychalinos

cpsychal@physics.upatras.gr

Ahmed S. Elwakil

elwakil@ieee.org

Ahmed G. Radwan

agradwan@ieee.org

Karabi Biswas

karabi@ee.iitkgp.ernet.in

1 Electronics Laboratory, Physics Department, University of Patras, 26504 Rio Patras, Greece

2 Electrical and Computer Engineering Department, College of Engineering, University of Sharjah, P.O. 27272, Sharjah, UAE

3 Nanoelectronics Integrated Systems Center (NISC), Nile University, 6th of October City, Egypt

4 Engineering Mathematics Department, Faculty of Engineering, Cairo University, Cairo, Egypt

5 Electrical Engineering Department, Indian Institute of Technology (IIT), Kharagpur, West Bengal 721302, India 
fractional-order systems as well as fractional-order circuit applications in renewable energy.

Below is a summary of the contributions under each of these themes.

\section{Circuit Theory of Fractional-Order Circuits}

In the paper "Minimum Energy Control of Fractional Positive Electrical Circuits with Bounded Inputs", the minimum energy control problem for the fractional positive electrical circuits with bounded inputs is formulated and solved. In the paper, "Analysis of the Transient State in a Series Circuit of the Class $R L_{\beta} C_{a}$ ", the authors present results of analysis of transient states in a series circuit of the class $R L_{\beta} C_{a}$. For this purpose, a method for determining the current and voltage waveforms in the analyzed circuit, based on the decomposition of rational functions into partial fractions, has been described. In the next paper, "An Iterative LMI Based Reduced Order Observer Design for Fractional Order Chaos Synchronization", an effort for designing a reduced-order observer for a nonlinear Lipschitz class of fractional-order systems has been performed. Sufficient conditions for stability of the observer, based on the Lyapunov technique, are derived and converted into linear matrix inequalities (LMIs). In the paper, "Improved Chaotic Dynamics of a Fractional Order System, its Chaos-suppressed Synchronization and Circuit Implementation", a new and improved fractional-order chaotic system (FOCS), design of controllers for suppression of chaos for commensurate and incommensurate orders in the regions: $0<\alpha \leq 1$ and $1<\alpha<2$, synchronization between the new FOCS (as master) and a nonidentical FOCS (as slave) when the master is in either chaotic mode or chaos-suppressed mode are introduced. The paper "Design and Performance Study of Dynamic Fractors in Any of the Four Quadrants" presents how a fractor can be realized, using RC-ladder circuit, meeting the predefined specifications on both fractance and exponent of the fractors. This work has employed GIC topology to realize fractors from any of the four quadrants, which means, fractors with exponents between -2 and +2. In the paper, "Three-Dimensional Chaotic Autonomous System with a Circular Equilibrium: Analysis, Circuit Implementation and its Fractional-Order Form", a three-dimensional autonomous chaotic system with a circular equilibrium is investigated. Some dynamical properties and behaviors of this system are described in terms of equilibria, eigenvalue structures, bifurcation diagrams, Lyapunov exponents, time series, and phase portraits. For specific parameters, the system displays periodic and chaotic attractors. Finally, the paper, "A Hybrid Optimization Based Approach for Parameter Estimation and Investigation of Fractional Dynamics in Ultracapacitors", aims at estimating the fractional model parameters of an ultracapacitor using experimental data and further investigating the dependency of fractional parameters on the operating conditions. The parameter estimation task has been formulated as an optimization problem which aims at minimizing the deviation between the model and experimental output using a hybrid optimization technique. The hybrid algorithm combines an improved version of seeker optimization algorithm for global exploration with a local search technique, namely the Nelder-Mead simplex search. 


\section{Fractional-Order Filter and Oscillator Design and Applications}

In the paper "Approximated Fractional-Order Inverse Chebyshev Lowpass Filters", a least-squares fitting routine is employed to approximate the stopband ripple characteristics of fractional-order Inverse Chebyshev low-pass filters which have fractional-order zeros and poles. Experimental results of a 1.5-order filter using approximated fractional-order capacitors in a Multiple-Input Biquad circuit validate the implementation. In the next paper, "Comparison of $(1+a)$ Fractional-Order Transfer Functions to Approximate Lowpass Butterworth Magnitude Responses", three fractional-order transfer functions are analyzed for differences in realizing $(1+a)$ order low-pass filters approximating a traditional Butterworth magnitude response. These transfer functions are realized by replacing traditional capacitors with fractionalorder capacitors in biquadratic filter topologies. Each fractional-order transfer function is realized using a Tow-Thomas biquad. The paper, "Practical Design and Evaluation of Fractional-Order Oscillator Using Differential Voltage Current Conveyors" deals with the design, analysis, computer simulation, and experimental measurement of fractional-order sinusoidal oscillator with two current conveyors, two resistors, and two fractional immittance elements. The conveyor used is based on the bulk-driven quasi-floating-gate (BD-QFG) technique in order to offer high threshold-to-supply voltage ratio and maximum input-to-supply voltage. The measurements taken on a real chip confirm the attractive features of the proposed oscillator. In the paper "On the Optimization of Fractional Order Low Pass Filters", three different optimization cases for normalized fractional-order low-pass filters (LPFs) with numerical, circuit, and experimental results are presented. A multi-objective optimization technique is used for controlling some filter specifications, which are the transition bandwidth, the stopband frequency gain and the maximum allowable peak in the filter passband. The extra degree of freedom provided by the fractional-order parameter allows the full manipulation of the filter specifications to obtain the desired response required by any application. In the next paper, "Asymptotic Solutions and Circuit Implementations of a Rayleigh Oscillator Including Cubic Fractional Damping Terms", a fractional-order Rayleigh oscillator model which involves a cubic damping term described by fractional derivatives is introduced. The presence of such a fractional damping term makes the analysis more difficult. A two-scale expansion method is employed for asymptotic solutions of the fractional-order Rayleigh oscillator, and an example is provided to compare the asymptotic solutions with the numerical solutions. The last paper, "Dual Estimation of Fractional Variable Order Based on the Unscented Fractional order Kalman Filter for Direct and Networked Measurements", is devoted to variable order estimation process when measurements are obtained in two different ways: directly and by lossy network. Since the problem of fractional-order estimation is highly nonlinear, dual estimation algorithm based on unscented fractional-order Kalman filter has been used. In the dual estimation process, state variable and order estimation have been divided into two subprocesses. For estimating the state variables and the fractional order, the fractional Kalman filter and the unscented fractional Kalman filter have been used, respectively. 


\section{Digital Circuits and Systems Approximating Fractional-Order Systems}

The aim of the paper "On Digital Realizations of Non-Integer Order Filters" is to present two efficient methods for digital realization of noninteger-order filters: discrete-time domain Oustaloup approximation and Laguerre impulse response approximation (LIRA). Properties of both methods are investigated with use of noninteger low-pass filter. Filters realized with presented methods are then used for filtering of EEG. In the next paper, "Introducing Fractional Order Dynamics to Sigma-Delta Modulators", an investigation of the performance of a fractional-order sigma-delta modulators wherein the integer order integrator is replaced by a fractional integrator of order $a(1<a<2)$ is carried out. A generalized approach to both linear frequencydomain and nonlinear time-domain modeling and characterization of fractional- order sigma-delta modulators is discussed. In the paper "Design of Wideband Fractional Order Differentiator Using Interlaced Sampling Method", the design of a wideband digital fractional- order differentiator (FOD) is investigated. First, conventional FOD designs are reviewed, and the reconstruction formula of the interlaced sampling method is used to design the proposed wideband FOD by index substitution and the Grünwald-Letnikov fractional derivative. The paper "Efficient Design of Discrete Fractional Order Differentiators Using Nelder-Mead Simplex Algorithm" presents a novel method to design fractional-order differentiator (FOD) operators through optimization using Nelder-Mead simplex algorithm (NMSA). For direct discretization, Al-Alaoui operator has been used. The numerator and the denominator terms of the resulting transfer function are further expanded using binomial expansion to a required order. The coefficients of $z$-terms in the binomial expansions are used as the starting solutions for the NMSA, and optimization is performed for a minimum magnitude root-mean-square error between the ideal and the proposed operator magnitude responses. In the paper "Design and Realization of Stand-Alone Digital Fractional Order PID Controller for Buck-Converter Fed DC Motor", a digital fractional-order proportional-integral derivative (FO-PID) controller for speed control of buck-converter fed DC motor is employed. Optimal pole-zero approximation method in discrete form is proposed for realization of digital fractional order controller. The stand-alone controller is implemented on embedded platform using the digital signal processor (DSP) TMS320F28027. In the last paper, "Optimal Design of 2-D FIR Filters with Quadrantally Symmetric Properties Using Fractional Derivative Constraints", an optimal design of two-dimensional finite impulse response (2D FIR) filters with quadrantally even symmetric impulse response using the fractional derivative constraints (FDCs) is presented. First, the design problem of 2D FIR filter is formulated as an optimization problem. Then, the fractional derivative constraints are imposed over the integral absolute error for the quadrantally even symmetric impulse response filter. Next, the optimized filter impulse response is computed using a hybrid optimization technique, called hybrid particle swarm optimization and gravitational search algorithm (HPSO-GSA). Further, the FDC values are also optimized, such that a flat passband and stopband response is achieved and the absolute L1-error is minimized. 


\section{Fractional-Order Circuit Applications in Renewable Energy}

The paper "Improvements in Dissolved Oxygen Control of an Activated Sludge Wastewater Treatment Process" shows a simple way of decreasing the operational costs, together with an increase in the performance and disturbance rejection by using fractional-order proportional-integral controllers on the dissolved oxygen concentration and on the air pressure generated by the air blower. Closed-loop system performance, using the fractional-order proportional-integral controller, is compared to those obtained by using the conventional proportional-integral controller on the dissolved oxygen concentration. They prove the robustness of the former controller against the parameters variations.

We earnestly hope that all the papers presented in this special issue will provide useful information for the readers of the CSSP Journal as well as for those who are interested in this highly interdisciplinary topic.

At this point, we would like to thank all the anonymous reviewers for their valuable assistance in selecting the best papers for this special issue. We also thank all the authors who have submitted their papers for this special issue. Special thanks go to the editor in chief of the CSSP Journal Prof. M.N.S. Swamy for his valuable support and assistance during the whole framework of this special issue.

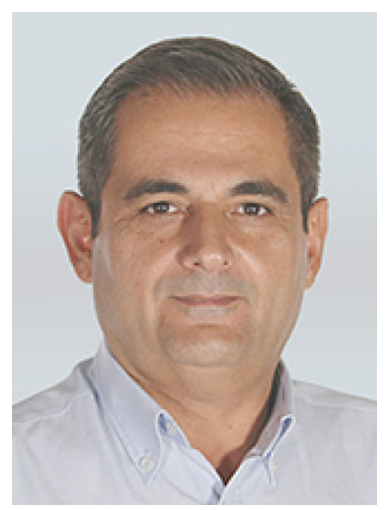

Costas Psychalinos received his B.Sc. and Ph.D. degrees in Physics and Electronics in the University of Patras, Greece, in 1986 and 1991, respectively. From 1993 to 1995, he worked as Postdoctoral Researcher with the VLSI Design Laboratory at the University of Patras. From 1996 to 2000, he was an Adjunct Lecturer with the Department of Computer Engineering and Informatics at the University of Patras. From 2000 to 2004, he was an Assistant Professor with the Electronics Laboratory, Department of Physics, Aristotle University of Thessaloniki, Greece. From 2004, he serves as faculty member in the Electronics Laboratory, Department of Physics, University of Patras, Greece, and currently, he is full professor. His research area is in the continuous- and discrete-time analog filtering, including companding filters, fractional-order filters, and in the development of ultra low-voltage building blocks for biomedical applications. He serves as member of the Editorial Board in the Analog Integrated Circuits and Signal Processing Journal and in the Microelectronics Journal, and as an Associate Editor in the Circuits Systems and Signal Processing Journal. He is also senior member of IEEE. 


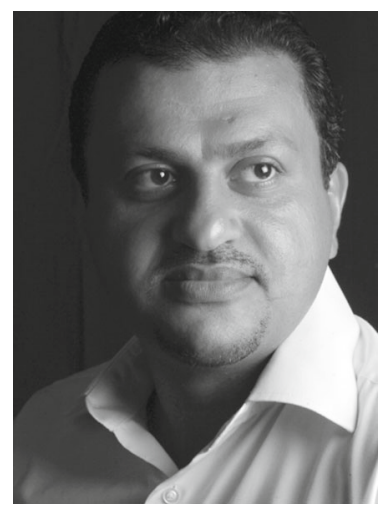

Ahmed S. Elwakil received the B.Sc. and M.Sc. in Electronics and Communications from Cairo University and his Ph.D. from the Department of Electrical and Electronic Engineering, National University of Ireland, University College Dublin. His research interests are primarily in the areas of Circuit Theory, Nonlinear Dynamics, Chaos Theory, as well as Fractional-Order Circuits and Systems with diverse applications ranging from the modeling of oscillatory networks and nonlinear electronic circuits to energy devices and biomaterials. He is the author and co-author of over 150 publications in these areas and currently serves as an Associate Editor for the Int. J. of Circuit Theory and Applications (Wiley). He is also an Associate Editor of the Int. J. Bifurcation \& Chaos, (World Scientific) and the Journal of Nonlinear Theory and its Applications (NOLTA). $\mathrm{He}$ is currently on the Editorial Board of the IEEE J. Emerging and Selected Topics in Circuits and Systems. Prof. Elwakil is an Associate Member of the Centre for Chaos Control and Synchronization at the City University of Hong Kong and the International Centre for Theoretical Physics. He was a keynote speaker at the sixth International Workshop on Chaos Fractals and Applications, Taiyuan, Shanxi, China, August 2013 and a keynote speaker at the Int. Conf. on Fractional Differentiation and Its Applications (ICFDA), Catania, Italy, June 2014, where he lectured on the theory of fractional-order circuits and systems. He has been a member of the IEEE Technical Committee on Nonlinear Circuits and Systems (TCNCAS) since 2003 and was a Lead Guest Editor for the IEEE J. of Emerging and Selected Topics in Circuits \& Systems, special issue on fractional-order circuits and systems (Sep. 2013) and a Guest Editor for a special issue on Chaos-Fractals Theories and Applications published in the J. of Mathematical Problems in Engineering, 2014. He was a visiting Professor/Researcher at Istanbul Technical University (Istanbul, Turkey), University of Calgary (Alberta, Canada), Queens University (Belfast, UK), Technical University of Denmark (Lyngby, Denmark), Ohio State University (Columbus, Ohio), University College Cork (Cork, Ireland), King Abdullah University of Science and Technology (KAUST, Saudi Arabia) and Nile University (Cairo, Egypt) where he is currently an Adjunct Prof. in the Nano-electronics Integrated Systems Center.

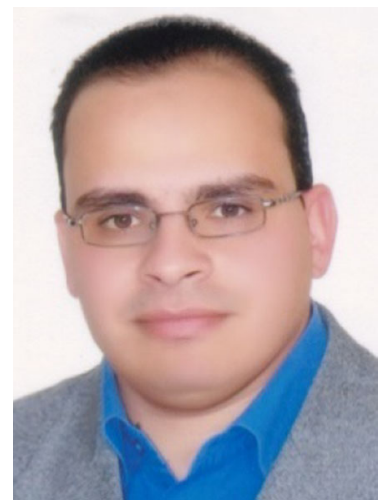

Ahmed G. Radwan received his B.Sc., M.Sc. and Ph.D. degrees from Cairo University, Cairo, Egypt, in 1997, 2002, and 2006 respectively. He is an Associate Professor in the Engineering Mathematics Department, Cairo University, Egypt, and Director of Nanoelectronics Integrated Systems Center (NISC), Nile University, Egypt. From 2008 to 2012, he joined the research teams at McMaster University, Canada and King Abdullah University of Science and Technology (KAUST), Saudi Arabia. He received the state achievements award for research in Mathematical Sciences in 2012, the Cairo University achievements award for research in Engineering Sciences in 2013, and best researcher award in Nile University in 2015, and organized many special sessions in different international conferences. His research area is in nonlinear circuit analysis, chaotic systems, fractional-order systems, and memristor-based circuits. He is the coauthor of more than 140 international papers, many international books, and six US Patents. He is a senior member of IEEE, Egyptian Academy of Sciences (EYAS), and Egyptian Center for the Advancement of Science, Technology and Innovation (ECASTI). 


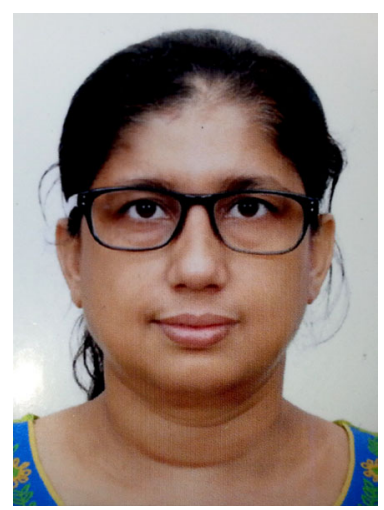

Karabi Biswas has received her B.Tech. and Ph.D. degree from I.I.T. Kharagpur in 1992 and 2007, respectively. She did her M.Tech. from Jadavpur University in 2000. She is presently Associate Professor in the department of Electrical Engineering, I.I.T. Kharagpur. Before that she served Jadavpur University as a faculty member in Instrumentation and Electronics Engineering. Her research interests include "Fractor development and study of fractional order systems". She also works on "Sensor development and Instrumentation system design". 